\title{
Next-generation sequencing of microRNAs reveals a unique expression pattern in different types of pituitary adenomas
}

\author{
Zongze $\mathrm{He}^{1)}$, Longyi Chen ${ }^{1)}$, Xiao $\mathrm{Hu}^{1)}$, Jian Tang ${ }^{1)}$, Linfu $\mathrm{He}^{2)}$, Junting $\mathrm{Hu}^{1)}$, Fan $\mathrm{Fei}^{1)}$ and Qi Wang ${ }^{1)}$ \\ 1) Department of Neurosurgery, Sichuan Academy of Medical Sciences and Sichuan Provincial People's Hospital, School of Medicine, \\ University of Electronic Science and Technology of China, Chengdu, Sichuan 610072, China \\ 2) Institute of Bioengineering, Jiangsu University, Zhenjiang, Jiangsu 212013, China
}

\begin{abstract}
Pituitary adenomas (PAs) are considered the most common intracranial tumor to cause serious morbidity because of dysregulated pituitary hormone secretions. Aberrant expression of microRNAs (miRNAs) is correlated with the development and function of the pituitary gland as well as the tumorigenesis of hypothalamic-pituitary axis-related pituitary tumors. In this study, we showed the differential expression patterns of miRNAs in NFPAs (nonfunctioning pituitary adenomas), GHPAs (growth hormone-secreting pituitary adenomas) and PRLPAs (prolactin-secreting pituitary adenomas) compared to those in three normal pituitary glands using the HiSeq 2000 sequencing system (Illumina). We validated miRNA expression using real-time quantitative polymerase chain reaction (RT-qPCR) analyses of samples from 73 patients (13 GHPAs, 42 NFPAs, and 18 PRLPAs) and 6 normal pituitary gland. We observed that miR-34c-3p was significantly downregulated in our PRLPA samples $(p<0.01)$, along with miR-34b-5p, miR-378 and miR-338-5p (all $p<0.05)$. In NFPAs, miR-493-5p was downregulated, and miR-181b-5p was significantly upregulated $(p<0.01)$. In GHPAs, miR-184 was significantly upregulated $(p<0.05)$. We observed that the tumor suppressive miR-124-3p was downregulated in both NFPAs and GHPAs. Taken together, we showed distinctive miRNA expression patterns in these three PAs, and these miRNA signatures in PA may have therapeutic potential as novel biomarkers for each type of PA.
\end{abstract}

Key words: Nonfunctioning pituitary adenomas, Growth hormone-secreting pituitary adenomas, Prolactin-secreting pituitary adenomas, miRNAs, RNA-sequencing

PITUITARY ADENOMAS (PAs) are one of the most common intracranial neoplasms, accounting for approximately $10 \%$ of intracranial tumors $[1,2]$. PAs can be further divided into non-functioning pituitary adenomas (NFPAs), which account for $\sim 40 \%$ of all PAs and functioning pituitary adenomas [3]. Functioning pituitary adenomas consist of growth hormone (GH)-producing pituitary adenoma, adrenocorticotropic hormone (ACTH)producing pituitary adenoma, prolactin (PRL)-producing

Submitted Nov. 9, 2018; Accepted Apr. 11, 2019 as EJ18-0487 Released online in J-STAGE as advance publication Apr. 26, 2019 Correspondence to: Qi Wang, Department of Neurosurgery, Sichuan Academy of Medical Sciences and Sichuan Provincial People's Hospital, School of Medicine, University of Electronic Science and Technology of China, No. 32 West Second Section First Ring Road, Chengdu 610072, Sichuan, China.

E-mail: wangqi@med.uestc.edu.cn

Correspondence to: Longyi Chen, Department of Neurosurgery, Sichuan Academy of Medical Sciences and Sichuan Provincial People's Hospital, School of Medicine, University of Electronic Science and Technology of China, No. 32 West Second Section First Ring Road, Chengdu 610072, Sichuan, China.

E-mail: chenly11@163.com pituitary adenoma, thyroid-stimulating hormone (TSH)producing pituitary adenoma, luteinizing hormone (LH)producing pituitary adenoma, and follicle-stimulating hormone (FSH)-producing pituitary adenoma [4]; in addition, these tumors may cause headache, visual disturbance, and hyperprolactinemia, among others [5]. PAs rarely transform into malignancies, and most are benign, but approximately $25 \%$ of tumors exhibit an invasive behavior [6]. The risks of radiation therapy and the limited effectiveness of surgery have resulted in an urgent need for new therapeutic options. Uncovering early diagnostic biomarkers is essential for the management of PAs. In addition, clarifying the molecular mechanisms of pituitary tumorigenesis is crucial for the development of new treatment options for PAs.

MicroRNAs (miRNAs) are a conserved family of small, non-coding RNA molecules that function as pivotal regulators of gene expression [7]. miRNAs modulate gene expression via binding the 3'-untranslated regions (3'-UTRs) of their target messenger RNAs (mRNAs), ultimately resulting in the repression of protein translation or mRNA decay [8,9]. Approximately $50 \%$ of 
miRNAs are in cancer-fragile genomic regions, suggesting that miRNAs affect tumor pathogenesis, progression, diagnosis, and prognosis [10-12]. Emerging data have demonstrated that miRNAs may act as oncogenes or tumor suppressors depending on the functions of their target genes [13]. Previous studies demonstrated that miRNAs play critical roles in pituitary tumorigenesis [14-16]. Deep sequencing is a technique that determines the precise order of nucleotides in a strand of DNA/ RNA. The advent of deep sequencing has substantially improved the quality of scientific discovery in many genomic research fields [17-19]. Darvasi et al. [20] found that the difference among high-throughput platforms is of great importance and selection of screening method can influence experimental results. And miRNA expression profiles could be different using various platforms [21].

miRNAs are emerging as the focus of pituitary tumor research [22]. The differential expression patterns of miRNAs have been extensively studied and show their roles in tumorigenesis and tumor suppression. Bottoni et al. [23] used a microarray to identify 30 miRNAs that are differentially expressed between normal and adenomatous pituitary samples and described histotype-specific miRNAs. Overexpression of miR-23a, miR-23b, and miR-24-2 and under-expression of miR-26b are characteristic of GHPAs and PRLPAs. The stable expression of miR-30a, miR-30b, and miR-30c was typical in ACTHproducing tumors. It was found that miR-26b can be used to distinguish between GHPAs and PRLPAs compared with NFPAs due to their high expression observed in NFPAs. Other signatures related to NFPAs include the downregulation of miR-127, miR-129, miR-134, and miR-203. Stilling et al. [24] reported that miR-122 and miR-493 were upregulated in pituitary carcinomas compared with that in adenomas.

miRNA expression profiles have also been shown to correlate with pituitary behaviors, miR-140 was found to be differentially expressed in nonfunctioning micro- and macro-adenomas. The expression of miR- $450 \mathrm{~b}$, miR424, miR-503, miR-542-3p, miR-629, and miR-214 correlates with tumor size in NFPAs [25]. ACTH-producing pituitary adenoma underexpressing miR-141 was found to have higher remission rates after surgical removal. Qian et al. [26] reported that adenomas that the expression level of let7 was significantly lower in invasive adenomas than in noninvasive adenomas, and potentially lower in high-grade (III, IV) adenomas than in low-grade (I, II) adenomas. In response to treatment with a dopamine agonist, treated $v s$. nontreated NFPAs showed differential expression of miR-134, miR-148, miR-155, miR-29b, miR-29c, and miR-200a. Other groups identified 13 miRNAs to be differentially expressed in so- matostatin analog-treated and nontreated GHPAs, and 7 miRNAs were linked to the response to drug treatment [27]. Regarding the functions of miRNAs, some target genes have been validated in PAs. Functional or experimental validation of miRNA-target interactions confirms the exact binding sites of miRNAs on the transcript; however, this process is labor intensive, as it requires cloning of the transcript region of interest into a vector before site-directed mutagenesis. Furthermore, protein samples are required to verify the effects of miRNAs on protein levels after miRNA transfection. miR-16-1 is under-expressed in GHPAs and PRLPAs [28]. An inverse correlation exists with arginyl-tRNA synthetase, one of their targets, that is linked to tumor growth. A previous study showed that the tumor suppressor Wee-1 kinase, which controls the cell cycle, was regulated by 3 overexpressed miRNAs: miR-128a, miR-155, and miR-516a-3p [29]. The high-mobility group A 1 (HMGA1) and HMGA 2 proteins were also found to be targeted by several miRNAs (let-7a, miR-15, miR-16, miR-26a, miR-196a2, miR-34b, miR-326, miR-432, miR-548c-3p, miR-570, and miR-603) in GH-/PRL-secreting adenomas $[26,30,31]$. Members of the HMGA family are nonhistone chromosomal proteins involved in cell growth, proliferation, differentiation, and death [32], and this family of proteins regulates numerous miRNAs that affect pituitary adenoma development. Pleiomorphic adenoma gene 1 (PLAG1), which induces cell cycle arrest and apoptosis, has also been suggested to be the target of overexpressed miR-26a in NFPAs. Transforming growth factor-b signaling was influenced by miRNAs in NFPAs, and 5 miRNAs showed a negative correlation with Smad3 expression [25], of which miR-140 was experimentally validated. In ACTHproducing pituitary adenoma, miR-26 was verified to have a role in regulating protein kinase $\mathrm{Cd}$ (PRKCD) and thus play an important role in the control of cell cycle of ACTH-producing pituitary tumor cells. However, these studies were not sufficient to identify miRNAs profiles in PAs.

Here, we demonstrate how to obtain miRNA sequencing reads from paired pituitary tumors and normal pituitary samples with deep sequencing experiments. The miRNA expression data from pituitary tumors and normal pituitary samples were statistically analyzed. A miRNA expression signature composed of differentially expressed miRNAs was found to clearly distinguish pituitary tumors from normal pituitary samples in hierarchical clustering analysis. Our study suggested a significant typical pattern of tumor suppressive miRNAs in the downregulation of multiple functioning pituitary adenomas. 


\section{Materials and Methods}

\section{Patients and subject recruitment}

The study was approved by the appropriate ethics review committees. Appropriate informed consent was obtained from all subjects. A total of 39 PA samples were surgically removed from 73 patients (13 GHPAs samples, 18 PRLPAs samples, and 42 NFPAs samples) who had undergone surgical resection at Sichuan Provincial People's Hospital between August 2016 and April 2017. Our subject group contained 35 females and 38 males with an average age of $51.3 \pm 14.0$. The subjects were all from Southwestern China provinces and predominantly Han Chinese with 4 Tibetans. The necessary information regarding the subjects with pituitary tumors recruited for this study is shown in Table 1 and Table 2. For sequencing, 3 samples of either GHPAs, PRLPAs or NFPAs were selected (detailed information are listed in the Supplementary Table 1). Normal human pituitary glands obtained from 6 autopsied patients deprived of endocrine diseases were used as controls. All tissue samples were collected with approval from all the patients and the ethics committee of Sichuan Provincial People's Hospital. After surgery, PAs and normal pituitary tissue were stored at $-80^{\circ} \mathrm{C}$ according to standard procedure. Some fragments of each sample were separated and immediately frozen in liquid nitrogen under RNase-free conditions until RNA extraction.

\section{$R N A$ extraction and next-generation sequencing}

Total RNA was extracted using a Trizol reagent (Invitrogen, Carlsbad, CA, USA) according to the manufacturer's protocol. Briefly, either the $3^{\prime}$ or 5' strand of RNAs was linked to adapters before reverse transcription using random hexamer primers for cDNAs (Truseq $^{\mathrm{TM}}$ Small RNA sample prep Kit, Illumina, San Diego, CA, USA). RNA product quality was assessed by the A260/ A280 ratio, and the RNA integrity was validated using the Agilent 2100 Bioanalyzer. miRNA libraries were constructed based on the manufacturer's standard protocol (Truseq ${ }^{\mathrm{TM}}$ Small RNA sample prep Kit) with modifications as previously reported [33]. The miRNA library was constructed and enriched after 11-12 PCR cycles and purified using 6\% Novex TBE (Tris-borate-EDTA)polyacrylamide gel electrophoresis (PAGE) (Invitrogen). Product yield was calculated using the TBS380 (Quant$\mathrm{iT}^{\mathrm{TM}}$ Picogreen, Invitrogen). For each miRNA library, 10 $\mu \mathrm{g}$ of total RNA was subjected to deep sequencing on the HiSeq 2000 sequencing system (Illumina) according to the manufacturer's standard protocol as previously described [34].
Table 1 Basic information of patients with pituitary tumors recruited in this study

\begin{tabular}{|c|c|c|c|c|}
\hline $\begin{array}{l}\text { Gender } \\
(\mathrm{M} / \mathrm{F})\end{array}$ & $\begin{array}{l}\text { Age } \\
\text { (year) }\end{array}$ & Ethnicity & Tumor Types & $\begin{array}{c}\text { Year of } \\
\text { Collection }\end{array}$ \\
\hline $\mathrm{F}$ & 74 & Han & GHPA & 2016 \\
\hline $\mathrm{F}$ & 52 & Han & GHPA & 2016 \\
\hline M & 44 & Han & GHPA & 2016 \\
\hline M & 41 & Han & GHPA & 2016 \\
\hline $\mathrm{F}$ & 53 & Han & GHPA & 2016 \\
\hline F & 26 & Han & GHPA & 2016 \\
\hline $\mathrm{F}$ & 48 & Han & GHPA & 2017 \\
\hline M & 65 & Han & GHPA & 2017 \\
\hline M & 53 & Han & GHPA & 2017 \\
\hline $\mathrm{F}$ & 53 & Han & GHPA & 2017 \\
\hline $\mathrm{F}$ & 53 & Han & GHPA & 2017 \\
\hline $\mathrm{F}$ & 31 & Han & GHPA & 2017 \\
\hline $\mathrm{F}$ & 44 & Han & GHPA & 2017 \\
\hline $\mathrm{F}$ & 75 & Han & NFPA & 2016 \\
\hline M & 51 & Han & NFPA & 2016 \\
\hline M & 64 & Han & NFPA & 2016 \\
\hline $\mathrm{F}$ & 42 & Han & NFPA & 2016 \\
\hline M & 67 & Han & NFPA & 2016 \\
\hline M & 78 & Han & NFPA & 2016 \\
\hline M & 54 & Han & NFPA & 2016 \\
\hline M & 71 & Han & NFPA & 2016 \\
\hline $\mathrm{F}$ & 70 & Han & NFPA & 2016 \\
\hline M & 55 & Han & NFPA & 2016 \\
\hline M & 58 & Han & NFPA & 2016 \\
\hline $\mathrm{F}$ & 47 & Han & NFPA & 2016 \\
\hline M & 64 & Han & NFPA & 2016 \\
\hline M & 71 & Han & NFPA & 2016 \\
\hline $\mathrm{F}$ & 54 & Han & NFPA & 2016 \\
\hline M & 23 & Han & NFPA & 2016 \\
\hline M & 46 & Tibetan & NFPA & 2016 \\
\hline M & 40 & Han & NFPA & 2016 \\
\hline $\mathrm{F}$ & 54 & Han & NFPA & 2016 \\
\hline $\mathrm{F}$ & 58 & Tibetan & NFPA & 2016 \\
\hline $\mathrm{F}$ & 44 & Han & NFPA & 2016 \\
\hline $\mathrm{F}$ & 58 & Han & NFPA & 2017 \\
\hline M & 68 & Han & NFPA & 2017 \\
\hline M & 51 & Han & NFPA & 2017 \\
\hline $\mathrm{F}$ & 50 & Tibetan & NFPA & 2017 \\
\hline $\mathrm{F}$ & 64 & Han & NFPA & 2017 \\
\hline F & 48 & Han & NFPA & 2017 \\
\hline
\end{tabular}


Table 1 Cont

\begin{tabular}{|c|c|c|c|c|}
\hline $\begin{array}{l}\text { Gender } \\
(\mathrm{M} / \mathrm{F})\end{array}$ & $\begin{array}{l}\text { Age } \\
\text { (year) }\end{array}$ & Ethnicity & Tumor Types & $\begin{array}{c}\text { Year of } \\
\text { Collection }\end{array}$ \\
\hline M & 74 & Han & NFPA & 2017 \\
\hline M & 30 & Han & NFPA & 2017 \\
\hline M & 47 & Han & NFPA & 2017 \\
\hline M & 29 & Han & NFPA & 2017 \\
\hline M & 51 & Han & NFPA & 2017 \\
\hline $\mathrm{F}$ & 51 & Han & NFPA & 2017 \\
\hline M & 65 & Han & NFPA & 2017 \\
\hline M & 49 & Han & NFPA & 2017 \\
\hline M & 53 & Han & NFPA & 2017 \\
\hline M & 68 & Han & NFPA & 2017 \\
\hline $\mathrm{F}$ & 58 & Han & NFPA & 2017 \\
\hline M & 67 & Han & NFPA & 2017 \\
\hline M & 25 & Han & NFPA & 2017 \\
\hline $\mathrm{F}$ & 45 & Han & NFPA & 2017 \\
\hline M & 47 & Han & NFPA & 2017 \\
\hline M & 31 & Han & PRLPA & 2016 \\
\hline $\mathrm{F}$ & 67 & Han & PRLPA & 2016 \\
\hline $\mathrm{F}$ & 49 & Han & PRLPA & 2016 \\
\hline $\mathrm{F}$ & 30 & Han & PRLPA & 2016 \\
\hline M & 48 & Han & PRLPA & 2016 \\
\hline $\mathrm{F}$ & 53 & Han & PRLPA & 2016 \\
\hline $\mathrm{F}$ & 67 & Han & PRLPA & 2016 \\
\hline $\mathrm{F}$ & 37 & Han & PRLPA & 2016 \\
\hline M & 32 & Han & PRLPA & 2016 \\
\hline $\mathrm{F}$ & 48 & Han & PRLPA & 2016 \\
\hline $\mathrm{F}$ & 25 & Tibetan & PRLPA & 2016 \\
\hline $\mathrm{F}$ & 64 & Han & PRLPA & 2017 \\
\hline M & 68 & Han & PRLPA & 2017 \\
\hline M & 32 & Han & PRLPA & 2017 \\
\hline M & 61 & Han & PRLPA & 2017 \\
\hline M & 29 & Han & PRLPA & 2017 \\
\hline $\mathrm{F}$ & 40 & Han & PRLPA & 2017 \\
\hline $\mathrm{F}$ & 37 & Han & PRLPA & 2017 \\
\hline
\end{tabular}

*M/F, Male/Female; GHPA, Growth hormone-secreting pituitary adenomas; NFPA, non-functioning pituitary adenomas; PRLPA, Prolactin-secreting pituitary adenomas.

\section{Sequencing data processing}

Small, non-coding RNA ( $<35 \mathrm{nt})$ expression was measured in three different types of PAs (GHPAs, NFPAs, and PRLPAs) and normal pituitary glands. A total of $218.53 \times 10^{6}$ raw reads were obtained by small
Table 2 Basic information of normal pituitary donors in this study

\begin{tabular}{ccccc}
\hline $\begin{array}{c}\text { Gender } \\
(\mathrm{M} / \mathrm{F})\end{array}$ & $\begin{array}{c}\text { Age } \\
\text { (year) }\end{array}$ & Ethnicity & $\begin{array}{c}\text { Post Mortem } \\
\text { Interval (hours) }\end{array}$ & $\begin{array}{c}\text { Year of } \\
\text { Collection }\end{array}$ \\
\hline M & 59 & Han & 6.5 & 2016 \\
M & 47 & Han & 7.0 & 2016 \\
F & 29 & Han & 21.5 & 2016 \\
F & 59 & Tibetan & 3.5 & 2017 \\
M & 43 & Han & 6.0 & 2017 \\
F & 71 & Tibetan & 4.5 & 2017 \\
\hline
\end{tabular}

*M/F, Male/Female.

RNA sequencing (error rate $\leq 0.015 \%$, Phred quality score of $20 \geq 99.0 \%$ ). There was no significant GCcontent bias in our RNA-sequencing data since each sample had a very similar GC content ranging from $51.23 \%$ to $53.48 \%$. Subsequently, low-quality reads were removed from the raw data, and the clean small, noncoding RNA reads were generated. A total of $7.58 \times 10^{6}$, $9.14 \times 10^{6}, 7.25 \times 10^{6}$ and $11.22 \times 10^{6}$ high-quality reads were obtained for the GHPAs, NFPAs, PRLPAs, and normal pituitary gland samples, respectively. The distribution of clean small RNA sequences according to their lengths was used for further type identification, and classification since the lengths of most small RNAs range from 18 to $40 \mathrm{nt}$ with slight differences. Our results showed that approximately $32 \%$ of the small RNA sequences we obtained were $22 \mathrm{nt}$ long, and $15 \%$ were $32 \mathrm{nt}$ long, suggesting that a significant portion of these sequences were miRNAs. As the length of miRNAs usually range from 21 to $22 \mathrm{nt}$, the lengths of siRNAs and piRNAs are typically $24 \mathrm{nt}$ and $30 \mathrm{nt}$, respectively.

Identical clean sequences were then merged into unique sequences to measure the abundance of small RNAs. The raw data were cleaned before small RNA classification and miRNA identification. The exclusion criteria included the following: 1) reads with no insertions due to the self-ligation of sequencing primers; 2) quality score less than $20 \mathrm{nt}$ on the 3' prime end; 3) reads with unknown nucleotides ("Ns"); and 4) reads with a length either less than $18 \mathrm{nt}$ or greater than $32 \mathrm{nt}$. We annotated the clean sequences in the Rfam public database (http://rfam.xfam.org/) and classified the small RNAs into different catalogs, such as miRNA, rRNA, tRNA, snRNA, ribozymes and other types of RNAs. We obtained 7,394,732 database matched-reads among a total of $7,734,007$ reads. The result suggested that miRNAs accounted for approximately $54.70 \%$ $(4,230,145 / 7,394,732)$ of the total reads, with $61.33 \%$ from $\mathrm{PA}$ and $52.55 \%$ from normal pituitary glands. Meanwhile, over $30 \%$ percent of the total sequenced 
small RNAs belong to the tRNA family $(2,366,733$ / $7,394,732)$. Ribozymes compromised less than $0.02 \%$ $(720 / 7,394,732)$ of the total sequenced small RNAs. It was evident that over half of the small RNAs sequenced could be classified into miRNA groups.

\section{Identification and analysis of known miRNAs}

We further identified these miRNAs using Bowtie, RNAfold, and miRdeep2 software according to the premature and mature miRNA sequence information in miRBase 21 (http://www.mirbase.org). The counts and transcripts per million (TPM) values for each known miRNA were collected and analyzed.

\section{Real-time quantitative polymerase chain reaction ( $R T-q P C R)$ validation}

The ten most dysregulated miRNAs were subjected to RT-qPCR analysis to validate the abnormal miRNAs discovered in NGS profiling. For each sample, $2 \mu \mathrm{g}$ of total RNA was reverse transcribed into cDNA using Moloney Murine Leukemia Virus Reverse Transcriptase (NEB, Ipswich, MA, USA) with a specific Stem-Loop RT Primer as previously mentioned [35]. RT-qPCR was performed using a Light Cycler (Roche, Basel, Switzerland) by the SYBR green method strictly according to the manufacturer's instruction. For each reaction, experiments were performed as follows: $20 \mu \mathrm{L}$ reaction volume containing $1 \mu \mathrm{L}$ of the reverse transcribed product, $10 \mu \mathrm{L}$ of $2 \times$ Mix SYBR Green I (Roche), $0.2 \mu \mathrm{L}$ of both the forward and reverse primer $(10 \mu \mathrm{M})$, and $8.6 \mu \mathrm{L}$ of RNase-free water. Three biological triplicates were prepared for each reaction, and U6 was chosen as the internal control. The relative change in the miRNA expression in each sample was compared to that in the normal pituitary sample using the $2^{-\Delta \Delta \mathrm{Ct}}$ method. The standard error of the mean was determined for each sample. The sequences of the primers used in this study are listed in Supplementary Table 2.

\section{Statistical analyses}

The RT-qPCR experiments were repeated independently at least 3 times. The data were expressed as the mean \pm standard deviation (SD). Multiple comparisons between groups were made by one-way analysis of variance (ANOVA) and Pearson's chi-square test, while group comparisons were carried out for quantitative experiments by Student's $t$-test. All statistical analyses were performed with SPSS software (SPSS, Chicago, IL, USA). For all tests, $p<0.05$ was considered statistically significant, and $p<0.01$ was considered highly significant.

\section{Results}

\section{Differentially-expressed miRNAs in prolactin- secreting pituitary adenomas}

As shown in Table 3, we obtained 18 upregulated (LogFC $\geq 3, p<0.05)$ miRNAs (miR-486-5p, miR148a-5p, miR-532-3p, miR-148a-3p, miR-135a-5p, miR96-5p, miR-200a-5p, miR-141-5p, miR-200c-3p, miR141-3p, miR-200a-3p, miR-429, miR-182-5p, miR-7-5p, miR-1251-5p, miR-1179, miR-7-2-3p, and miR-200b$3 p)$ and 35 downregulated ( $\operatorname{LogFC} \leq-3, p<0.05)$ miRNAs (miR-375, miR-548i, miR-9-3p, miR-9-5-p, miR-767-5p, miR-183-5p, miR-183-3p, miR-92b-3p, miR-100-3p, miR-2355-5p, miR-124-3p, miR-218-5p, miR-34c-3p, miR-548ab, miR-584-5p, miR-1249-3p, miR-625-3p, miR-34c-5p, miR-34b-5p, miR-365a-3p, miR-365b-3p, miR-338-5p, miR-330-3p, miR-219a2-3p, miR-193b-3p, miR-338-3p, miR-330-5p, miR-577, miR-181b-5p, miR-6716-3p, miR-222-3p, miR-100-5p, miR-181a-3p, miR-219a-5p, miR-181a-5p, and miR570) in our sequencing data. The hierarchical clustering heatmap of these 53 differentially expressed miRNAs in PRLPAs are shown in Fig. 1A. Subsequently, we validated the top 7 most differentially expressed miRNAs in 17 samples of PRLPAs. Our results suggested that miR-34c-3p was significantly downregulated in our PRLPAs $(p<0.01)$, along with miR-34b-5p, miR-378 and miR-338-5p (all $p<0.05)$. However, we observed no significant differences $(p>0.05)$ in miR-7-5p, miR200a-5p or miR-141-5p expression between the PRLPAs and normal pituitary sample groups (Fig. $1 \mathrm{~B}$ to $1 \mathrm{H}$ ). We compared our data with the microarray datasets in the GEO (Gene Expression Omnibus) database and found that 2 miRNAs (miR-34c-3p, miR-338-5p) were significantly downregulated in either non-aggressive (LogFC $\geq 3, p<0.05)$ or aggressive PRLPAs (LogFC $\geq 3, p<$ 0.05 ) from the dataset GSE46294 (4 aggressive PRLPAs, 8 non-aggressive PRLPAs and 2 normal pituitary samples); this finding is consistent with our RT-qPCR results, indicating that miR-34c-3p and miR-338-5p may play a vital role in regulating the function of PRLPAs. Previous studies also showed that miR-34b was drastically and constantly down-regulated in GHPAs [30].

\section{Differentially expressed miRNAs in nonfunctioning pituitary adenomas}

In NFPA samples, we observed 16 upregulated miRNAs (miR-181a-3p, miR-181a-5p, miR-181b-5p, miR-10a-5p, miR-7-5p, miR-148a-3p, miR-1185-1-3p, miR-200c-3p, miR-429, miR-375, miR-200b-3p, miR141-3p, miR-7-2-3p, miR-200a-5p, miR-1179, and miR-200a-3p) (Log FC $\geq 3, p<0.05$ ) and 14 downregulated miRNAs (miR-338-5p, miR-338-3p, miR-34c-5p, 
Table 3 Numbers of significantly changed miRNAs between different comparisons

\begin{tabular}{lcccc}
\hline Comparison & Direction & $\begin{array}{c}p<0.05 \text { and } \\
|\operatorname{logFC}|>1\end{array}$ & $\begin{array}{c}p<0.05 \text { and } \\
|\operatorname{logFC}|>3\end{array}$ & $\begin{array}{c}p<0.05 \text { and } \\
|\operatorname{logFC}|>5\end{array}$ \\
\hline GHPA vs. Normal & up & 74 & 65 & 16 \\
& down & 48 & 30 & 14 \\
PRLPA $v$ s. Normal & up & 26 & 18 & 16 \\
& down & 38 & 35 & 11 \\
NFPA $v$ vs. Normal & up & 14 & 16 & 10 \\
& down & 16 & 14 & 12 \\
\hline
\end{tabular}

${ }^{\#} \operatorname{LogFC}$ represents Log2 values of relative fold change between pituitary adenomas and normal samples. *Up represents up-regulation and down represents down-regulation in the each group.

A

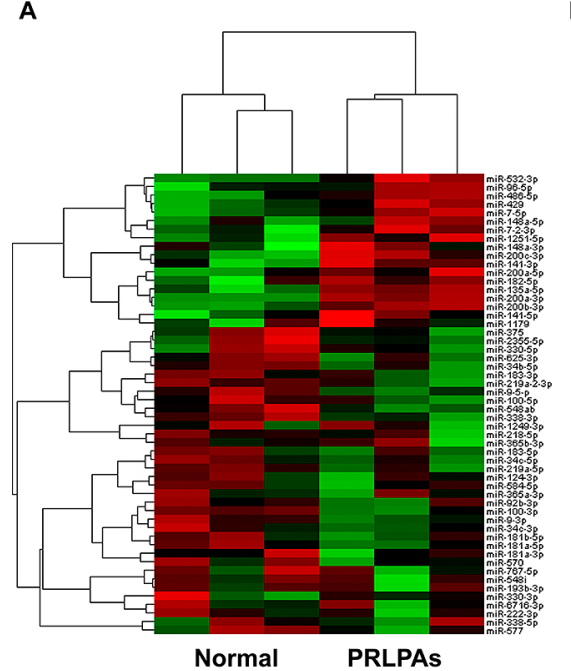

F

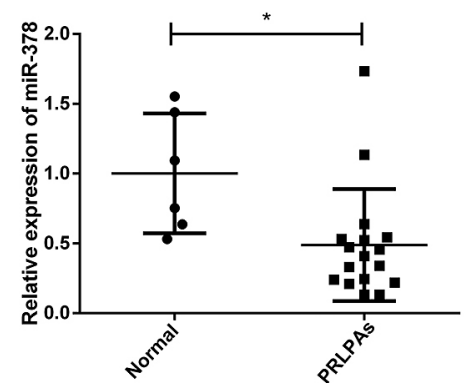

B

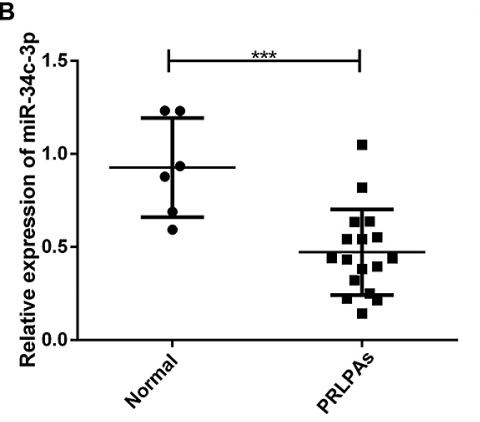

D

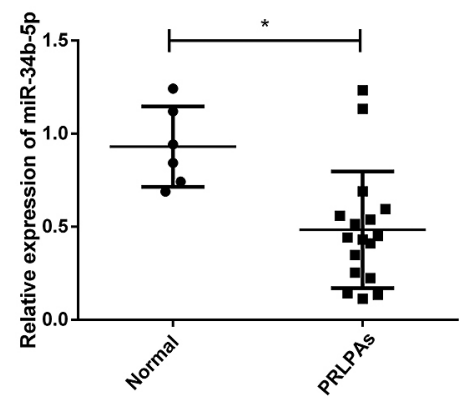

G

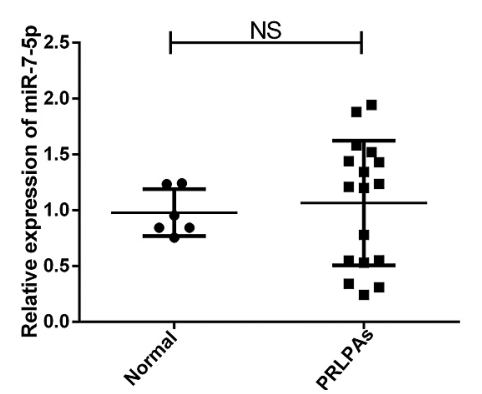

C
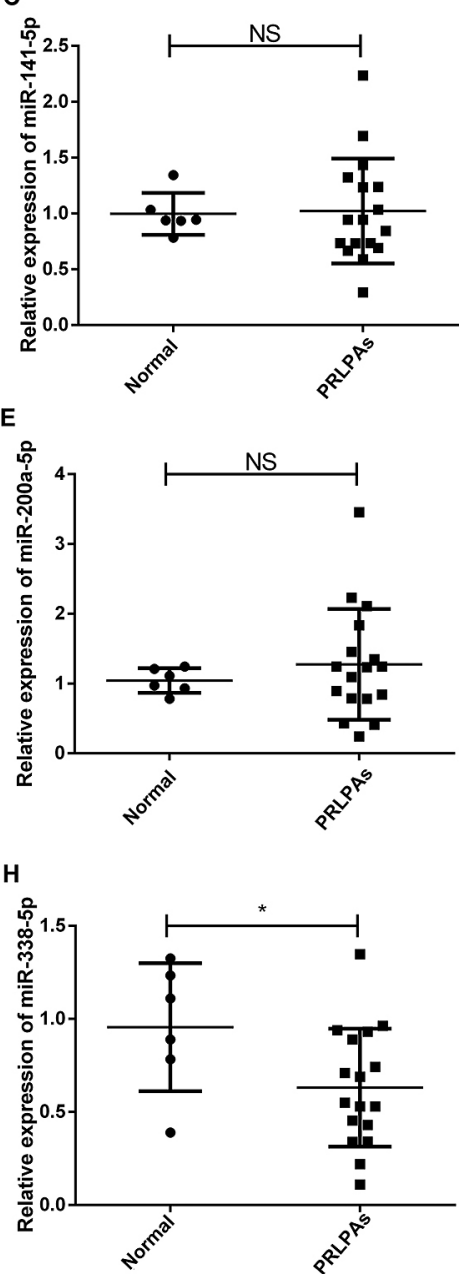

Fig. 1 Hierarchical clustering and RT-qPCR analysis validation of differentially expressed microRNAs between prolactin-secreting pituitary adenomas (PLRPAs) and control normal pituitary autopsy samples. (A) Hierarchical clustering heatmap of most differentially expressed miRNAs in PRLs. (B-H) RT-qPCR results of miR-34c-3p (B), miR-141-5p (C), miR-34b-5p (D), miR-200a-5p (E), miR-378 (F), miR-7-5p (G), miR-338-5p (H) expressions in the PRLPAs $(n=17)$ and normal pituitary sample groups $(n=6)$. The one-way ANOVA was used for the comparison between groups. NS: no significance, $*(p<0.05), * * *(p<$ $0.01)$ 
A

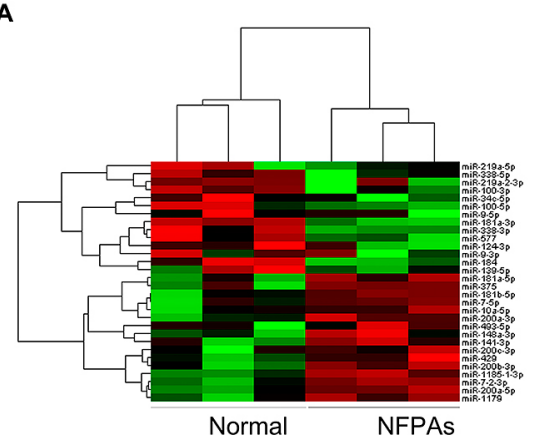

D

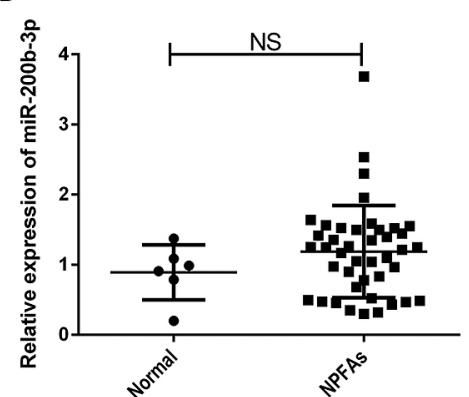

B

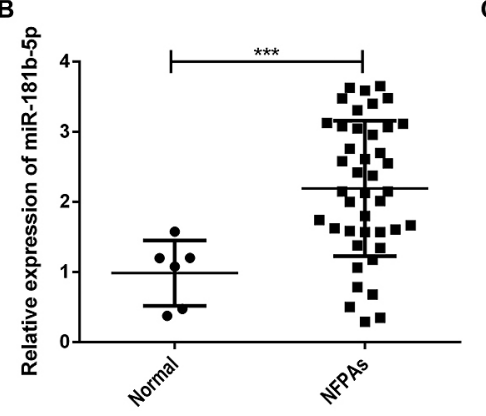

E

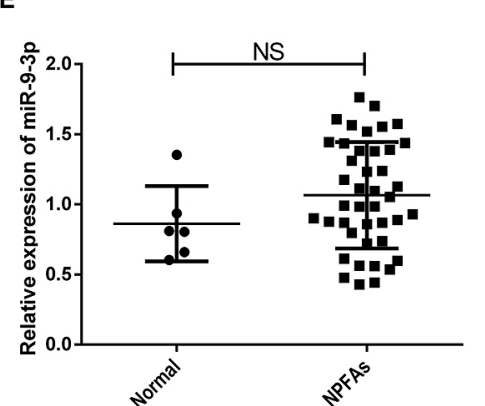



$\mathbf{F}$

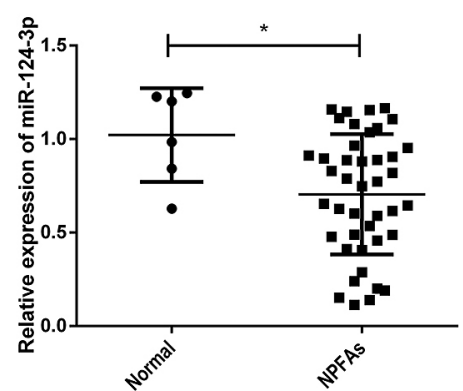

Fig. 2 Hierarchical clustering and RT-qPCR analysis validation of differentially expressed microRNAs between nonfunctioning pituitary adenomas (NFPAs) and control normal pituitary autopsy samples. (A) Hierarchical clustering heatmap of most differentially expressed miRNAs in NFPAs. (B-F) RT-qPCR results of miR-181b-5p (B), miR-493-5p (C), miR-200b-3p (D), miR-9-3p (E), miR-124-3p (F), expressions in the NFPAs $(n=42)$ and normal pituitary sample groups $(n=6)$. The one-way ANOVA was used for the comparison between groups. NS: no significance, $*(p<0.05), * * *(p<0.01)$

miR-184, miR-100-5p, miR-219a-5p, miR-493-5p, miR219a-2-3p, miR-577, miR-9-3p, miR-9-5p, miR-124-3p, miR-100-3p, and miR-139-5p) (Log FC $\leq-3, p<0.05)$ (Table 3, Fig. 2A). Subsequent validation of the top 5 most differentially expressed miRNAs in 42 NFPA adenomas samples showed that miR-493-5p and miR124-3p were downregulated $(p<0.05)$ (Fig. 2C, 2F), while miR-181b-5p was significantly upregulated $(p<$ 0.01 ) (Fig. 2B). However, our results indicated that there were no significant differences $(p>0.05)$ in miR-200b$3 p$ or miR-9-3p expression between the NFPA and normal pituitary sample groups (Fig. 2D, 2E).

\section{Differentially expressed miRNAs in growth hormone-secreting pituitary adenomas}

In GHPA samples, we identified 65 upregulated miRNAs and 30 downregulated miRNAs (Table 3, Fig. $3 \mathrm{~A})$. Subsequent validation of the top 6 most differentially expressed miRNAs in 13 GHPAs showed that miR-184 was upregulated $(p<0.05)$ (Fig. 3B), while miR-124-3p was downregulated $(p<0.05)$ (Fig. 3E). However, our results indicated that there were no significant differences in miR-584-5p, miR-100-5p, miR409-3p and miR-181b-3p expression between the GHPAs and normal pituitary sample groups $(p>0.05)$ (Fig. 3C, 3D, 3F and 3G).

\section{Discussion}

In this study, we generated miRNA expression profiles for GHPAs, PRLPAs, and NFPAs against normal pituitary tissues using NGS and RNA deep sequencing technology. We found 122 differentially expressed miRNAs in GHPAs and 70 differentially expressed miRNAs in PRLPAs. Furthermore, 30 miRNAs in NFPAs exhibited significant gene expression differences $(p<0.05$ and fold change $>2$ ), and 20 miRNAs (17 downregulated and 3 upregulated) common to all adenoma histotypes showed potential to be molecular biomarkers (Table 4). Then, select miRNAs were validated by qRT-PCR.

We validated that 3 tumor suppressive miRNAs (miR-34c, miR-338-5p and miR-375) were downregulated in PRLPAs. The tumor suppressive roles of miR-34c and miR-338 have been reported in multiple cancers. For instance, miR-338-5p downregulation in esophageal squamous cell carcinoma enhances radiosensitivity by targeting survival pathways to induce apoptosis [36]. MiR-375 inhibits tumor growth in hepatocellular carcinoma, esophageal squamous cell carcinoma and gastric cancer [37-39]. miR-34c can suppress tumor growth and metastasis in nasopharyngeal carcinoma [40]. However, the functions of these miRNAs were never studied in PRLPAs. 
A

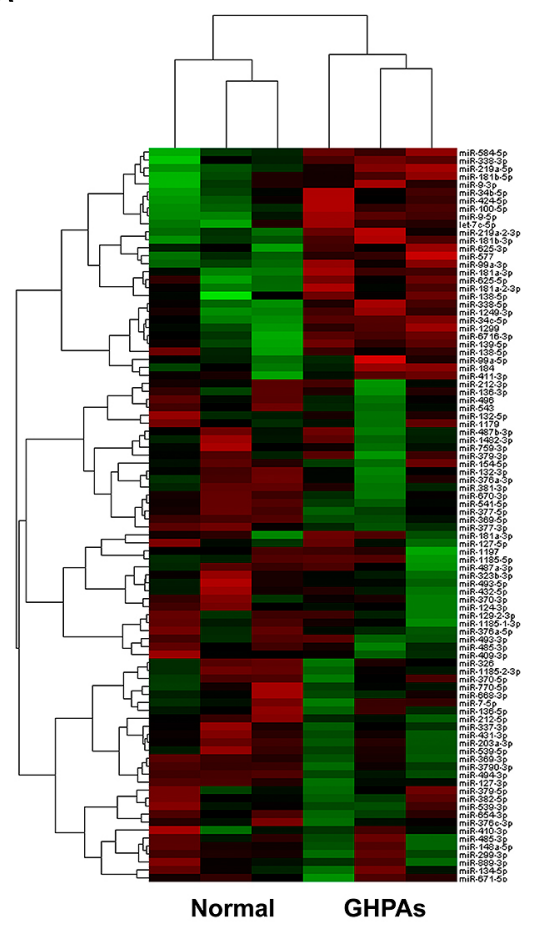

B

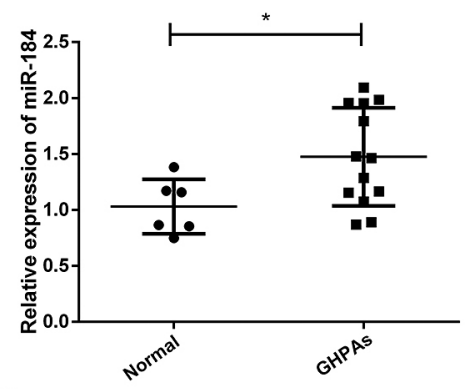

D

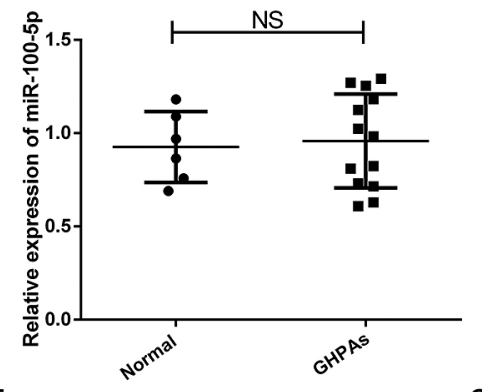

F
官 1.6

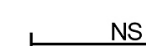

NS

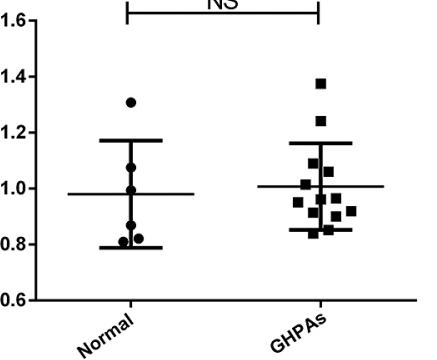

C

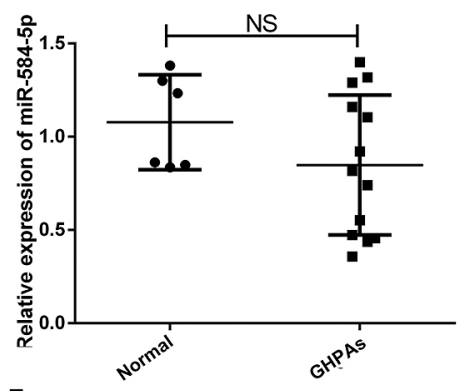

E

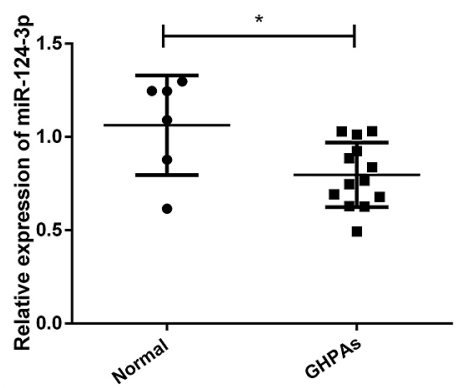

G



Fig. 3 Hierarchical clustering and RT-qPCR analysis validation of differentially expressed microRNAs between growth hormonesecreting pituitary adenomas (GHPAs) and control normal pituitary autopsy samples. (A) Hierarchical clustering heatmap of most differentially expressed miRNAs in GHPAs. (B-G) RT-qPCR results of miR-184 (B), miR-584-5p (C), miR-100-5p (D), miR-124-3p (E), miR-181b-3p (F), miR-409-3p (G) expressions in the GHPAs $(n=13)$ and normal pituitary sample groups $(n=$ $6)$. The one-way ANOVA was used for the comparison between groups. NS: no significance, $*(p<0.05)$

As for NFPAs, 16 miRNAs are upregulated, and 14 are downregulated. Of them, miR-493-5p and miR124-3p were significantly downregulated while miR$181 \mathrm{~b}-5 \mathrm{p}$ was upregulated. Wu et al. [41] also reported four upregulated (miRNAs hsa-miR-181b-5p, hsa-miR181d, hsa-miR-191-3p, and hsa-miR-598) and two downregulated miRNAs (hsa-miR-3676-5p and hsamiR-383) in NFPA samples. By performing Gene Ontology (GO) and Kyoto Encyclopedia of Genes and Genomes (KEGG) pathway analyses, they demonstrate that these differentially-expressed miRNAs are related to a series of biological processes and several signaling pathways [41]. Thus, these miRNAs may play a role in modulating the processes in NFPA progression, particularly miR-181b-5p, which needs further functional and mechanistic validations.

Regarding GHPAs, miR-184 was significantly upregulated while miR-124-3p was downregulated in GHPAs.
Another group has also reported that miR-124 was downregulated in GHPAs samples [27]. Visvanathanet et al. [42] demonstrated that SCP1 (small C-terminal domain phosphatase 1) played an anti-neural role during CNS development. miR-124 can inhibit SCP1 expression by directly targeting SCP1-3'UTR. Thus, miR-124 may contribute to CNS development since timely downregulation of SCP1 is critical for the induction of neurogenesis. However, similar as those in PRLPAs, the functions of these miRNAs need further investigation.

NGS sequencing technology provides a better global view of abnormal miRNA expression profiles than previous methodologies, a finding that is further verified by RT-qPCR. For the first time, we show that 10 miRNAs are abnormally expressed in three types of pituitary adenomas, and these putative miRNA targets contained a serial of oncogenes like BRAF, TWIST1, and ERBB2, suggesting an alternation of critical oncogenic pathways 
Table 4 Signifcantly dysregulated microRNAs of GHPA, PRLPA, NFPA adenomas vs. normal pituitary

\begin{tabular}{lccc}
\hline Unique ID & $\begin{array}{c}\text { logFC } \\
(\text { GHPA/Normal })\end{array}$ & $\begin{array}{c}\text { logFC } \\
\text { (PRLPA/Normal) }\end{array}$ & $\begin{array}{c}\text { logFC } \\
\text { (NFPA/Normal) }\end{array}$ \\
\hline hsa-miR-219a-2-3p & -9.09 & -8.88 & -9.21 \\
hsa-miR-219a-5p & -8.47 & -9.46 & -8 \\
hsa-miR-9-3p & -7.01 & -7.66 & -8.68 \\
hsa-miR-9-5p & -5.82 & -6.97 & -8.39 \\
hsa-miR-124-3p & -5.09 & -8.32 & -6.23 \\
hsa-miR-100-5p & -6.83 & -6.15 & -6.37 \\
hsa-miR-34c-5p & -7.47 & -4.81 & -6.45 \\
\hline hsa-miR-181a-3p & -6.51 & -6.03 & -6.09 \\
hsa-miR-100-3p & -7.29 & -5.59 & -5.32 \\
\hline hsa-miR-181b-5p & -6.47 & -5.31 & -6.03 \\
hsa-miR-181a-5p & -5.14 & -4.36 & -5.82 \\
hsa-miR-1249-3p & -5.28 & -4.21 & -4.91 \\
hsa-miR-338-5p & -4.3 & -4.99 & -4.32 \\
hsa-miR-625-3p & -3.09 & -4.02 & -3.76 \\
hsa-miR-767-5p & -2.85 & -3.69 & -4.11 \\
hsa-miR-625-5p & -3.29 & -2.74 & -4.28 \\
hsa-miR-181a-2-3p & -3.9 & -1.93 & -4.03 \\
hsa-miR-7-2-3p & 3.4 & 5.38 & 3.48 \\
hsa-miR-1179 & 4.3 & 5.46 & 4.07 \\
hsa-miR-148a-3p & 6.52 & 3.98 & 4.71 \\
\hline
\end{tabular}

"LogFC represents $\log 2$ values of relative fold change between pituitary adenomas and normal samples. The $p$ value in significance analysis and FDR value (false discovery rate) of all shown data are less than $5 \%$.

Table 5 Putative target genes of differentially expressed miRNAs

\begin{tabular}{lcl}
\hline miRNA & Chromosome location & \multicolumn{1}{c}{ Putative target } \\
\hline hsa-miR-219a-2-3p & $9 \mathrm{q} 34.11$ & TEAD1, PAFAH1B1, ITGB1BP1, ASPH, PROM1 \\
\hline hsa-miR-219a-5p & $6 \mathrm{p} 21.32$ & TGFBR2, SCN7A, CDH1, REPS2, SMAD2 \\
hsa-miR-9-3p & $15 \mathrm{q} 26.1$ & ONECUT2, DPYSL3, CDH1, MCC, INTS6 \\
\hline hsa-miR-9-5p & $15 \mathrm{q} 26.1$ & NT5C3, ONECUT2, CLSPN, NDEL1, TRIM25 \\
hsa-miR-124-3p & $8 \mathrm{p} 23.1$ & SCN7A, PAFAH1B1, JDP2, BIRC5, IL16 \\
hsa-miR-100-5p & $11 \mathrm{q} 24.1$ & NT5C3, DNA2, ST5, GPR55, MTOR \\
hsa-miR-34c-5p & $11 \mathrm{q} 23.1$ & ATP5A1, AHI1, TGFBR2, CDH1, ERBB2 \\
hsa-miR-181a-3p & $1 \mathrm{q} 32.1$ & AHI1, DNA2, ATP5A1, PCSK6, BRAF \\
hsa-miR-7-2-3p & $15 \mathrm{q} 26.1$ & AGO1, CDC27, ZNF551, FAXC, DUT \\
hsa-miR-1179 & $15 \mathrm{q} 26.1$ & LIFR, PDZD2, TWIST1, NUAK1, ZNF592 \\
\hline
\end{tabular}

in all types of pituitary adenomas (Table 5 ). Our results revealed multiple miRNAs in PA as potential tumor biomarker candidates for pituitary adenomas.

\section{Acknowledgments}

We thank all the patients and their families for participating in this study. This research project was supported by a grant from the Department of Science and Technology of Sichuan Province, China (no. 2016FZ0092). 


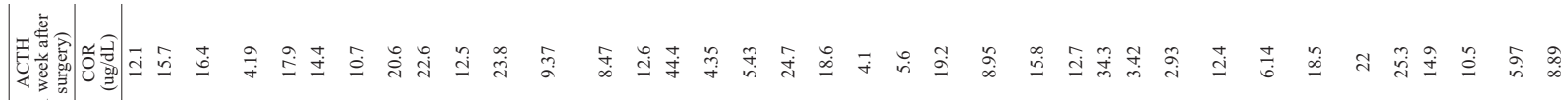

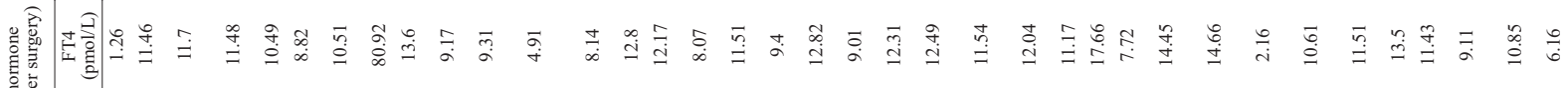

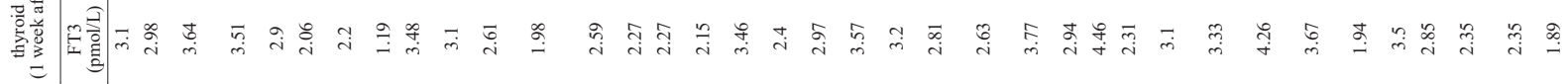



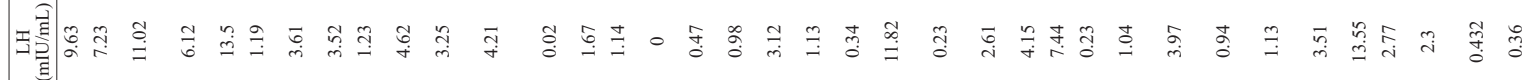

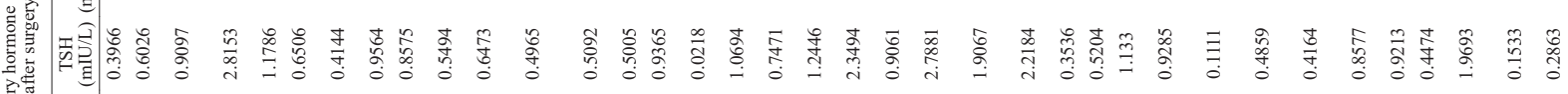

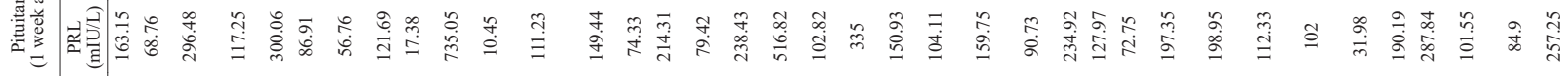

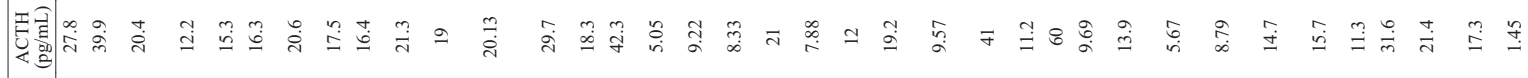

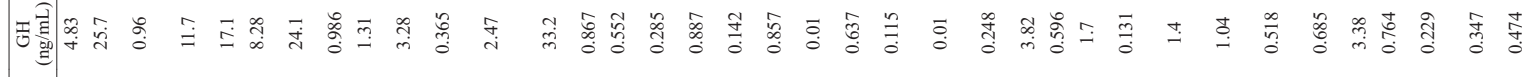

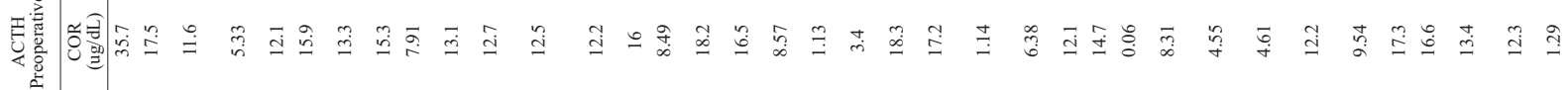

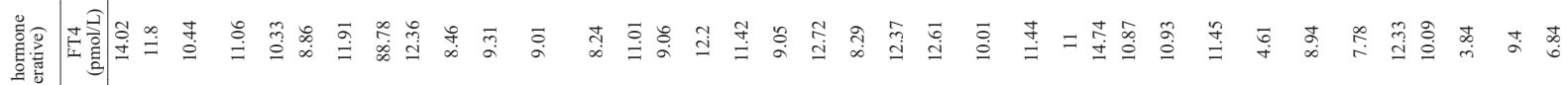

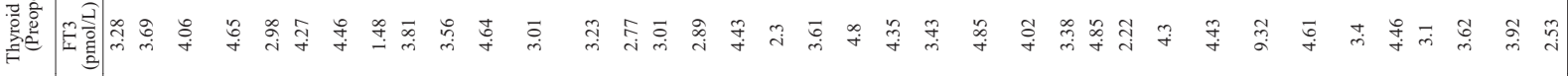

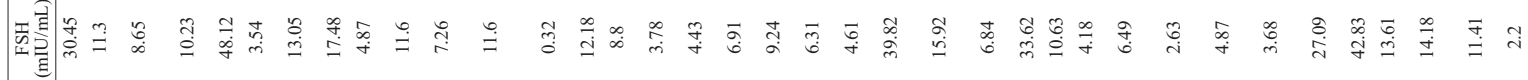

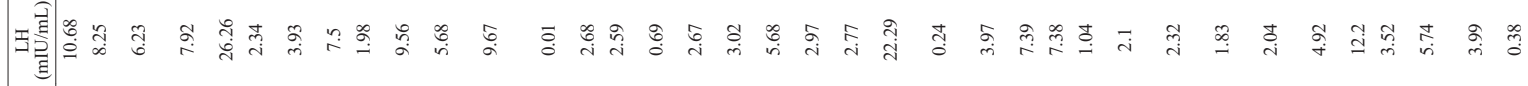

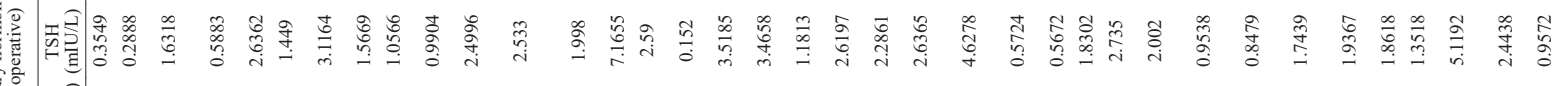

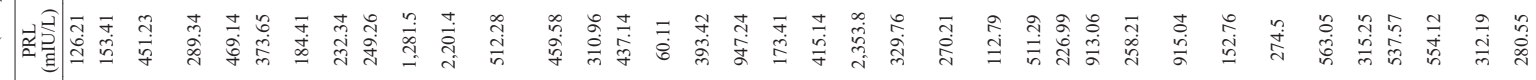

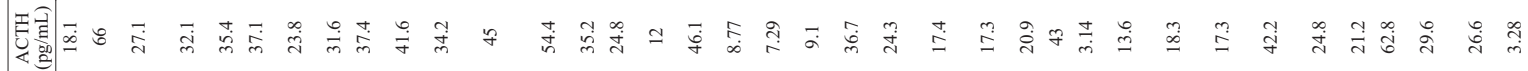

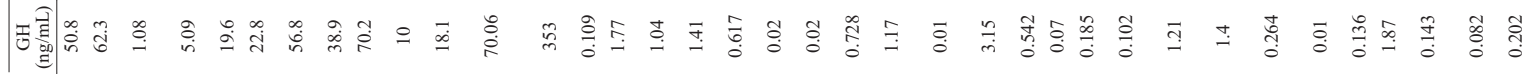

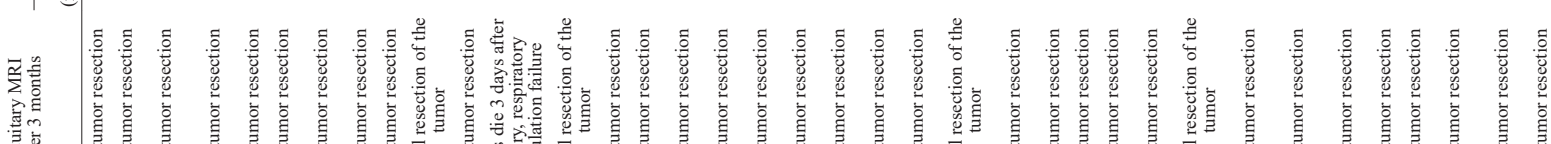

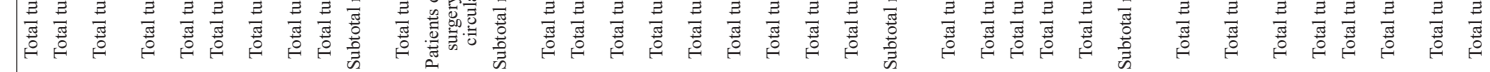

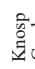

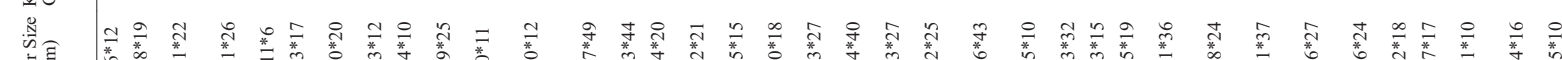

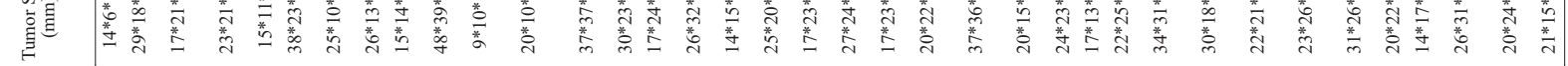

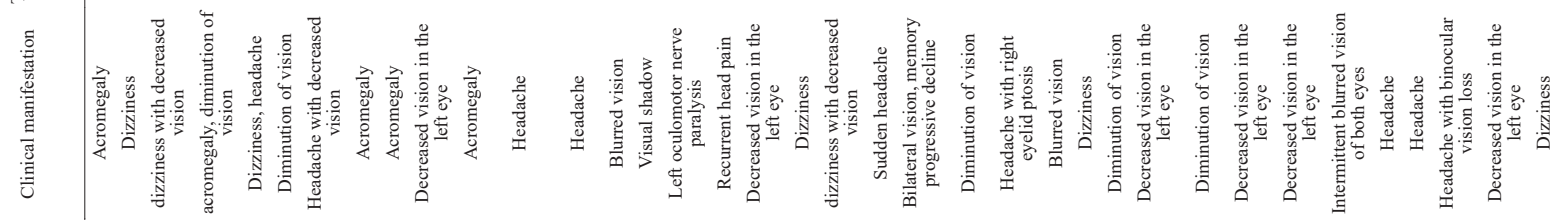

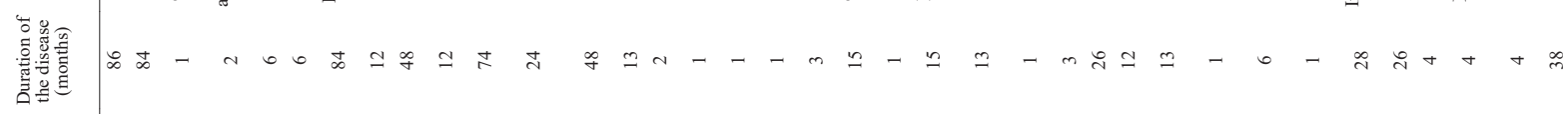



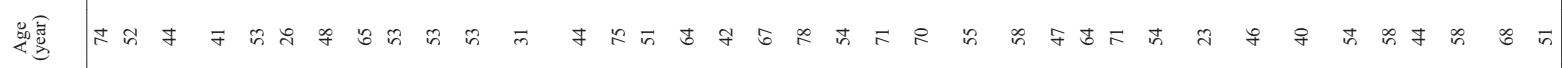

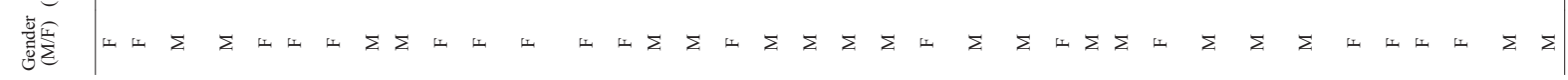




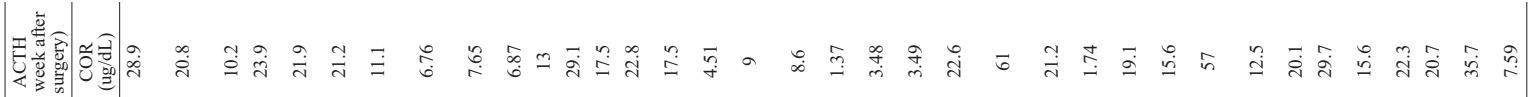

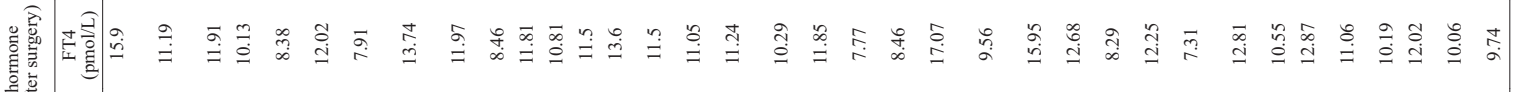

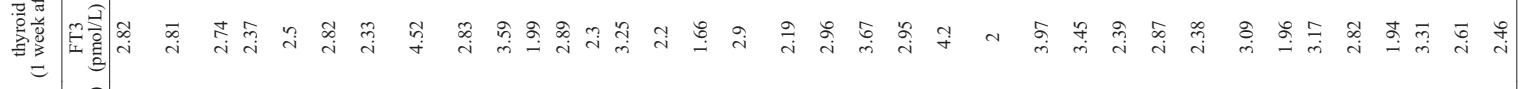

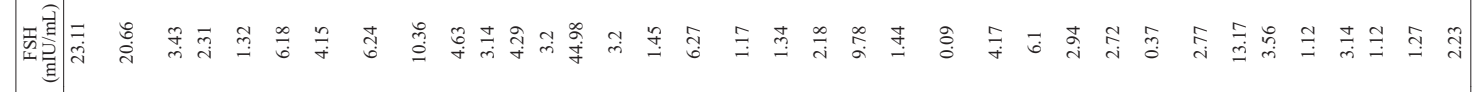

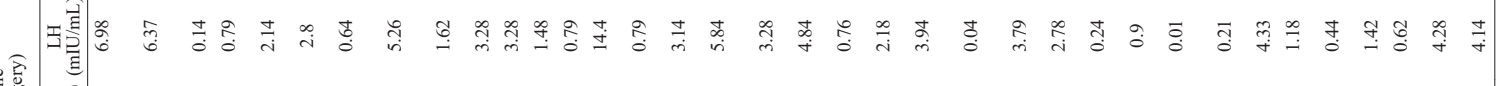

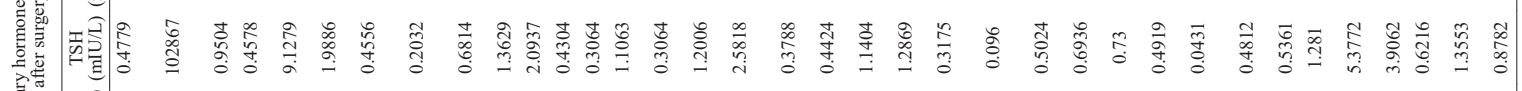

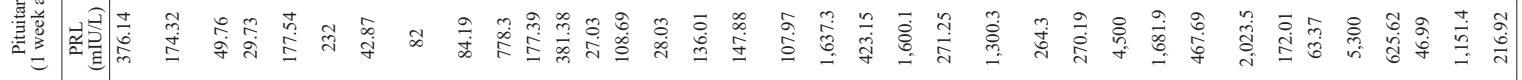

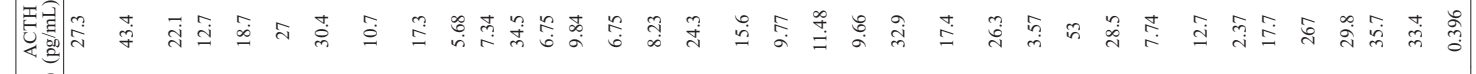



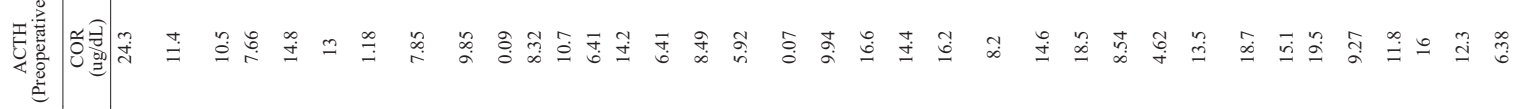

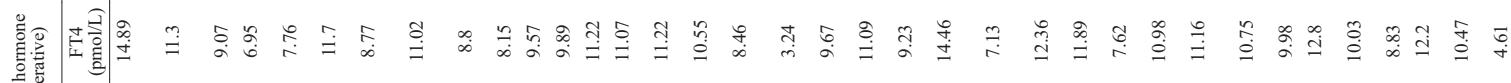

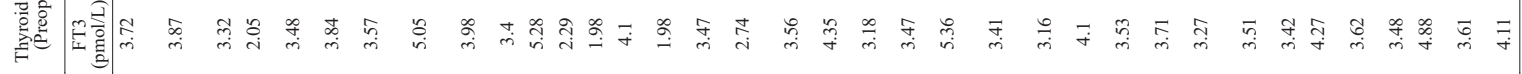

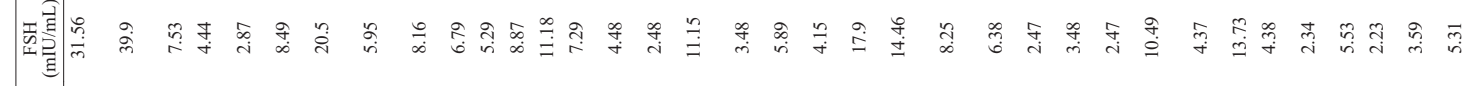

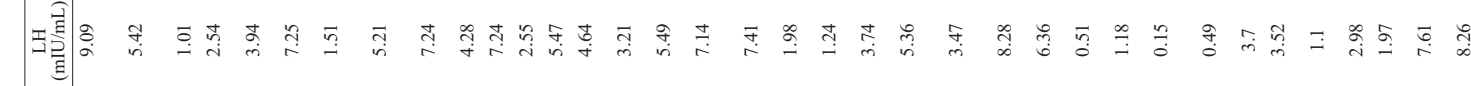

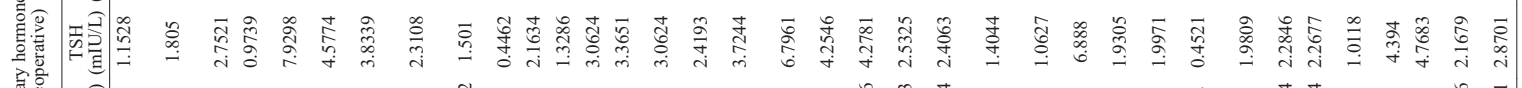

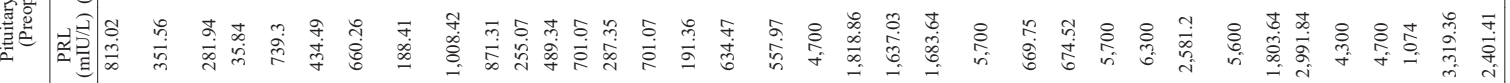

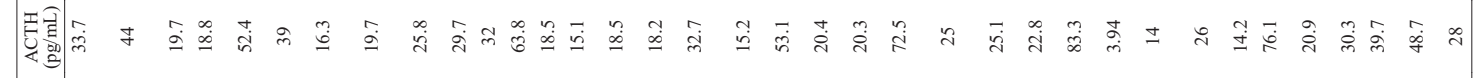

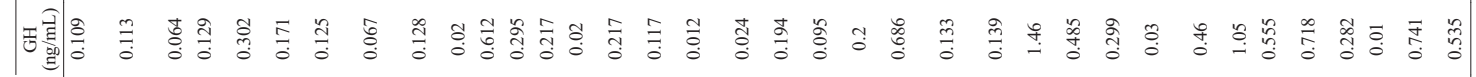

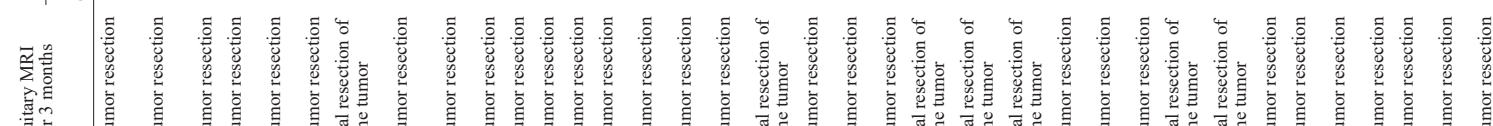

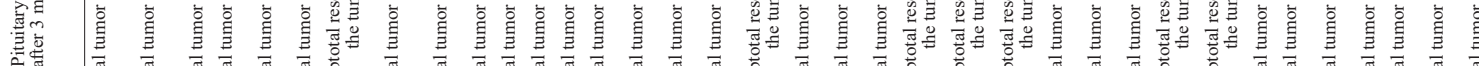

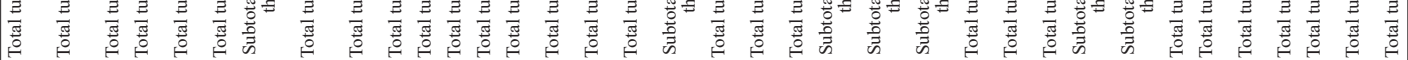

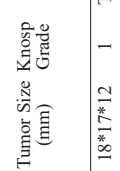

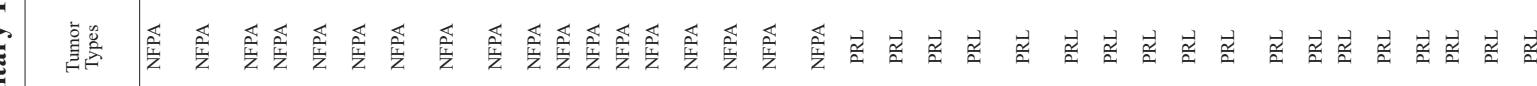


Supplementary Table 2 The Primer Sequence of miRNAs

\begin{tabular}{|c|c|}
\hline Name & Sequence \\
\hline hsa-miR-34c-3p-RT & GTCGTATCCAGTGCGTGTCGTGGAGTCGGCAATTGCACTGGATACGACCCTGGC \\
\hline hsa-miR-34c-3p-F & GCCAATCACTAACCACACG \\
\hline hsa-miR-141-5p-RT & GTCGTATCCAGTGCGTGTCGTGGAGTCGGCAATTGCACTGGATACGACTCCAAC \\
\hline hsa-miR-141-5p-F & GCGCATCTTCCAGTACAGT \\
\hline hsa-miR-34b-5p-RT & GTCGTATCCAGTGCGTGTCGTGGAGTCGGCAATTGCACTGGATACGACCAATCA \\
\hline hsa-miR-34b-5p-F & GCCTAGGCAGTGTCATTAGC \\
\hline hsa-miR-200a-5p-RT & GTCGTATCCAGTGCGTGTCGTGGAGTCGGCAATTGCACTGGATACGACTCCAGC \\
\hline hsa-miR-200a-5p-F & GCATCTTACCGGACAGTG \\
\hline hsa-miR-378-RT & GTCGTATCCAGTGCGTGTCGTGGAGTCGGCAATTGCACTGGATACGACCCTTCT \\
\hline hsa-miR-378-F & GCCACTGGACTTGGAGTC \\
\hline hsa-miR-7-5p-RT & GTCGTATCCAGTGCGTGTCGTGGAGTCGGCAATTGCACTGGATACGACACAACA \\
\hline hsa-miR-7-5p-F & GCCGTGGAAGACTAGUGATTT \\
\hline hsa-miR-338-5p-RT & GTCGTATCCAGTGCGTGTCGTGGAGTCGGCAATTGCACTGGATACGACCACTCA \\
\hline hsa-miR-338-5p-F & GCAACAATATCCTGGTGC \\
\hline hsa-miR-200b-5p-RT & GTCGTATCCAGTGCGTGTCGTGGAGTCGGCAATTGCACTGGATACGACTCCAAT \\
\hline hsa-miR-200b-5p-F & GCATCTTACTGGGCAGC \\
\hline hsa-miR-181b-5p-RT & GTCGTATCCAGTGCGTGTCGTGGAGTCGGCAATTGCACTGGATACGACAACCCA \\
\hline hsa-miR-181b-5p-F & AACATTCATTGCTGTCGG \\
\hline hsa-miR-493-5p-RT & GTCGTATCCAGTGCGTGTCGTGGAGTCGGCAATTGCACTGGATACGACAATGAA \\
\hline hsa-miR-493-5p-F & GCCTTGTACATGGTAGGCT \\
\hline hsa-miR-9-3p-RT & GTCGTATCCAGTGCGTGTCGTGGAGTCGGCAATTGCACTGGATACGACACTTTC \\
\hline hsa-miR-9-3p-F & GCCGGATAAAGCTAGATAACC \\
\hline hsa-miR-124-3p-RT & GTCGTATCCAGTGCGTGTCGTGGAGTCGGCAATTGCACTGGATACGACGGCATT \\
\hline hsa-miR-124-3p-F & GTAAGGCACGCGGTG \\
\hline hsa-miR-184-RT & GTCGTATCCAGTGCGTGTCGTGGAGTCGGCAATTGCACTGGATACGACACCCTT \\
\hline hsa-miR-184-F & GCTGGACGGAGAACTGAT \\
\hline hsa-miR-584-5p-RT & GTCGTATCCAGTGCGTGTCGTGGAGTCGGCAATTGCACTGGATACGACCTCAGT \\
\hline hsa-miR-584-5p-F & GTTATGGTTTGCCTGGG \\
\hline hsa-miR-100-5p-RT & GTCGTATCCAGTGCGTGTCGTGGAGTCGGCAATTGCACTGGATACGACCACAAG \\
\hline hsa-miR-100-5p-F & GAACCCGTAGATCCGAA \\
\hline hsa-miR-181b-3p-RT & GTCGTATCCAGTGCGTGTCGTGGAGTCGGCAATTGCACTGGATACGACTTGCAT \\
\hline hsa-miR-181b-3p-F & GCGCTCACTGAACAATGA \\
\hline hsa-miR-409-3p-RT & GTCGTATCCAGTGCGTGTCGTGGAGTCGGCAATTGCACTGGATACGACAGGGGT \\
\hline hsa-miR-409-3p-F & GGAATGTTGCTCGGTGA \\
\hline hsa-miR-R & CAGTGCGTGTCGTGGA \\
\hline U6-F & CTCGCTTCGGCAGCACA \\
\hline U6-R & AACGCTTCACGAATTTGCGT \\
\hline
\end{tabular}




\section{References}

1. Ntali G, Wass JA (2018) Epidemiology, clinical presentation and diagnosis of non-functioning pituitary adenomas. Pituitary 21: 111-118.

2. Gittleman H, Ostrom QT, Farah PD, Ondracek A, Chen Y, et al. (2014) Descriptive epidemiology of pituitary tumors in the United States, 2004-2009. J Neurosurg 121: 527535.

3. Shao S, Li X (2013) Clinical features and analysis in 1,385 Chinese patients with pituitary adenomas. J Neurosurg Sci 57: 267-275.

4. Melmed S (2015) Pituitary tumors. Endocrinol Metab Clin North Am 44: 1-9.

5. Giustina A (2012) Keep an eye on nonfunctioning pituitary adenomas. Clin Endocrinol (Oxf) 77: 656-657.

6. Ezzat S, Asa SL (2006) Mechanisms of disease: the pathogenesis of pituitary tumors. Nat Clin Pract Endocrinol Metab 2: 220-230.

7. Jiang X, Zhang X (2013) The molecular pathogenesis of pituitary adenomas: an update. Endocrinol Metab (Seoul) 28: 245-254.

8. Bartels CL, Tsongalis GJ (2009) MicroRNAs: novel biomarkers for human cancer. Clin Chem 55: 623-631.

9. Fabian MR, Sonenberg N, Filipowicz W (2010) Regulation of mRNA translation and stability by microRNAs. Annu Rev Biochem 79: 351-379.

10. Slaby O, Krekac D, Hrstka R, Svoboda M, Vyzula R (2008) Involvement of microRNAs in cancer biology and possibilities of their application to diagnostic and predictive oncology. Cas Lek Cesk 147: 25-31 (In Czech).

11. Ke TW, Wei PL, Yeh KT, Chen WT, Cheng YW (2015) MiR-92a promotes cell metastasis of colorectal cancer through PTEN-mediated PI3K/AKT pathway. Ann Surg Oncol 22: 2649-2655.

12. Krol J, Loedige I, Filipowicz W (2010) The widespread regulation of microRNA biogenesis, function and decay. Nat Rev Genet 11: 597-610.

13. Pang JC, Kwok WK, Chen Z, Ng HK (2009) Oncogenic role of microRNAs in brain tumors. Acta Neuropathol 117: 599-611.

14. Leone V, Langella C, D'Angelo D, Mussnich P, Wierinckx A, et al. (2014) Mir-23b and miR-130b expression is downregulated in pituitary adenomas. Mol Cell Endocrinol 390: 1-7.

15. Renjie W, Haiqian L (2015) MiR-132, miR-15a and miR-16 synergistically inhibit pituitary tumor cell proliferation, invasion and migration by targeting Sox5. Cancer Lett 356: 568-578.

16. He W, Huang L, Li M, Yang Y, Chen Z, et al. (2017) MiR-148b, MiR-152/ALCAM axis regulates the proliferation and invasion of pituitary adenomas cells. Cell Physiol Biochem 44: 792-803.

17. Morozova O, Marra MA (2008) Applications of nextgeneration sequencing technologies in functional genomics. Genomics 92: 255-264.

18. Davey JW, Hohenlohe PA, Etter PD, Boone JQ, Catchen
JM, et al. (2011) Genome-wide genetic marker discovery and genotyping using next-generation sequencing. Nat Rev Genet 12: 499-510.

19. Teer JK (2014) An improved understanding of cancer genomics through massively parallel sequencing. Transl Cancer Res 3: 243-259.

20. Darvasi O, Szabo PM, Nemeth K, Szabo K, Spisak S, et al. (2019) Limitations of high throughput methods for miRNA expression profiles in non-functioning pituitary adenomas. Pathol Oncol Res 25: 169-182.

21. Git A, Dvinge H, Salmon-Divon M, Osborne M, Kutter C, et al. (2010) Systematic comparison of microarray profiling, real-time PCR, and next-generation sequencing technologies for measuring differential microRNA expression. RNA 16: 991-1006.

22. Wierinckx A, Roche M, Legras-Lachuer C, Trouillas J, Raverot G, et al. (2017) MicroRNAs in pituitary tumors. Mol Cell Endocrinol 456: 51-61.

23. Bottoni A, Zatelli MC, Ferracin M, Tagliati F, Piccin D, et al. (2007) Identification of differentially expressed microRNAs by microarray: a possible role for microRNA genes in pituitary adenomas. $J$ Cell Physiol 210: 370-377.

24. Stilling G, Sun Z, Zhang S, Jin L, Righi A, et al. (2010) MicroRNA expression in ACTH-producing pituitary tumors: up-regulation of microRNA-122 and -493 in pituitary carcinomas. Endocrine 38: 67-75.

25. Butz H, Liko I, Czirjak S, Igaz P, Korbonits M, et al. (2011) MicroRNA profile indicates downregulation of the TGFbeta pathway in sporadic non-functioning pituitary adenomas. Pituitary 14: 112-124.

26. Qian ZR, Asa SL, Siomi H, Siomi MC, Yoshimoto K, et al. (2009) Overexpression of HMGA2 relates to reduction of the let-7 and its relationship to clinicopathological features in pituitary adenomas. Mod Pathol 22: 431-441.

27. Mao ZG, He DS, Zhou J, Yao B, Xiao WW, et al. (2010) Differential expression of microRNAs in GH-secreting pituitary adenomas. Diagn Pathol 5: 79.

28. Bottoni A, Piccin D, Tagliati F, Luchin A, Zatelli MC, et al. (2005) miR-15a and miR-16-1 down-regulation in pituitary adenomas. $J$ Cell Physiol 204: 280-285.

29. Butz H, Liko I, Czirjak S, Igaz P, Khan MM, et al. (2010) Down-regulation of Wee1 kinase by a specific subset of microRNA in human sporadic pituitary adenomas. $J$ Clin Endocrinol Metab 95: E181-E191.

30. D'Angelo D, Palmieri D, Mussnich P, Roche M, Wierinckx A, et al. (2012) Altered microRNA expression profile in human pituitary GH adenomas: down-regulation of miRNA targeting HMGA1, HMGA2, and E2F1. J Clin Endocrinol Metab 97: E1128-E1138.

31. Palmieri D, D'Angelo D, Valentino T, De Martino I, Ferraro A, et al. (2012) Downregulation of HMGAtargeting microRNAs has a critical role in human pituitary tumorigenesis. Oncogene 31: 3857-3865.

32. Cleynen I, Van de Ven WJ (2008) The HMGA proteins: a myriad of functions (Review). Int J Oncol 32: 289-305. 
33. Buermans HPJ, Ariyurek Y, van Ommen G, den Dunnen JT, t'Hoen PAC (2010) New methods for next generation sequencing based microRNA expression profiling. $B M C$ Genomics 11: 716 .

34. Tong XH, Xu B, Zhang YW, Liu YS, Ma CH (2014) Research resources: comparative microRNA profiles in human corona radiata cells and cumulus oophorus cells detected by next-generation small RNA sequencing. PLoS One 9: e106706.

35. Chen C, Ridzon DA, Broomer AJ, Zhou Z, Lee DH, et al. (2005) Real-time quantification of microRNAs by stemloop RT-PCR. Nucleic Acids Res 33: e179.

36. Park M, Yoon HJ, Kang MC, Kwon J, Lee HW (2017) MiR-338-5p enhances the radiosensitivity of esophageal squamous cell carcinoma by inducing apoptosis through targeting survivin. Sci Rep 7: 10932.

37. Kinoshita T, Hanazawa T, Nohata N, Okamoto Y, Seki N (2012) The functional significance of microRNA-375 in human squamous cell carcinoma: aberrant expression and effects on cancer pathways. J Hum Genet 57: 556-563.
38. Ding L, Xu Y, Zhang W, Deng Y, Si M, et al. (2010) MiR-375 frequently downregulated in gastric cancer inhibits cell proliferation by targeting JAK2. Cell Res 20: 784-793.

39. He XX, Chang Y, Meng FY, Wang MY, Xie QH, et al. (2012) MicroRNA-375 targets AEG-1 in hepatocellular carcinoma and suppresses liver cancer cell growth in vitro and in vivo. Oncogene 31: 3357-3369.

40. Li YQ, Ren XY, He QM, Xu YF, Tang XR, et al. (2015) MiR-34c suppresses tumor growth and metastasis in nasopharyngeal carcinoma by targeting MET. Cell Death Dis 6: e1618.

41. Wu S, Gu Y, Huang Y, Wong TC, Ding H, et al. (2017) Novel biomarkers for non-functioning invasive pituitary adenomas were identified by using analysis of microRNAs expression profile. Biochem Genet 55: 253-267.

42. Visvanathan J, Lee S, Lee B, Lee JW, Lee SK (2007) The microRNA miR-124 antagonizes the anti-neural REST/ SCP1 pathway during embryonic CNS development. Genes Dev 21: 744-749. 